\title{
Projection Methods With Different Trial and Test Spaces*
}

\author{
By M. S. Mock
}

\begin{abstract}
We consider finite element projection methods for linear partial differential equations, in which the spaces of trial functions and test functions may be different. In addition to approximation and smoothness properties, conditions implying equality of dimensions and uniform coerciveness are required, the most important of which resembles a strong form of an inverse assumption.

Our results provide a mechanism for the difference in the rate of convergence of Galerkin procedures with cubic splines and Hermite cubics, applied to first order symmetric hyperbolic problems [13].
\end{abstract}

1. Introduction. In this paper we discuss the approximate solution of linear wellposed partial differential equations, using finite element projection methods in which the trial space, in which an approximate solution is obtained, may be different from the space of test functions. Perhaps the best known of such methods are the collocation schemes, which have been applied to two point boundary value problems in [4], [5], [23] and to parabolic problems in one space variable in [7]. Higher order methods utilizing different spaces have also been applied to one-dimensional problems in [4], [18], [22].

The requirement that the trial and test spaces have equal dimension is an important restriction on such methods. Equality of dimension is implied by the coerciveness conditions of [2]. In one dimension, the coerciveness conditions can be satisfied by taking one of the spaces (usually the test space, but the trial space in [22]) to be the image of the other under an ordinary differential operator of even order. This mapping is made elliptic by requiring the domain space to be conforming. This technique has recently been generalized to higher dimensions in [10], using an elliptic partial differential operator as the mapping between spaces.

In the following, we consider an abstract one-parameter family of elliptic pseudodifferential operators as the mapping between the two spaces. (A background reference for such operators is [29].) The coerciveness conditions are formulated as requirements on this family of operators; indeed, our basic results for elliptic and parabolic problems, Theorems 1 and 2 below, could be alternatively obtained by showing that for sufficiently small mesh size, our schemes are variational principles in the sense of [2]. Our results contain some examples of methods considered in [2], [10], [12], [18], [22], [27] as special cases.

Application to nonlinear elliptic problems was the motivation for this work.

Received September 16, 1974; revised June 2, 1975.

AMS (MOS) subject classifications (1970). Primary 65N35; Secondary 65M15.

* Results obtained at the Courant Institute of Mathematical Sciences, New York University, with the United States Air Force, Contract AF-F44620-74-C-0062. 
Nonlinear problems can require more smoothness of the trial functions than linear problems of the same order, if for no other reason than for the discrete problem to be properly defined. Such problems are typically formulated, however, as that of finding a fixed point of a compact (nonlinear) operator [15], [16]. A nonlinear elliptic equation may assume the identity minus compact form on a relatively high Sobolev space, and this is the space from which an approximate solution is obtained. In [19], we show that for finite element methods, it suffices that the approximate solution satisfy the boundary conditions in the same sense as for linear problems; but we have not been able to relax the smoothness requirements.

In order to simplify the discrete equations requiring solution in such problems, it seems desirable for the test functions to have their support in as small a region as possible. Thus we are motivated to consider nonlocal operators between the trial and test space. Unfortunately, our analysis does not include collocation schemes, but does include schemes intermediate between collocation and Galerkin methods. The machinery we develop has also been applied in some subsequent work on hyperbolic problems [20]

The best known examples of spaces for which our methods are applicable are the smooth splines; other possibilities are discussed in Section 6. For smooth spline spaces, stronger results than ours are obtained for the heat equation in [26] and for periodic initial value problems in [27]. Also for one-dimensional problems, better results are obtained in [9] and references there cited.

2. Elliptic and Parabolic Problems. We first consider the problem

$$
L u=f, \quad x \in R^{n},
$$

in which $L$ is a uniformly and strongly elliptic positive definite operator of order $2 m$ with smooth coefficients, $f$ is a specified function in $H^{k-2 m}\left(R^{n}\right) \equiv H^{k-2 m}, k$ a positive integer, and $u$ is the desired solution in $H^{k}$. Our results can be readily extended to include the case of indefinite invertible $L$ by the methods of [15], [24]. In the following, we use ( , ) for the real scalar product over $R^{n}, C$ for a generic constant and $\|\cdot\|_{j}$ for the $H^{j}$ norm,

$$
\|\phi\|_{j}^{2}=\int_{R_{\xi}^{n}}|\hat{\phi}(\xi)|^{2}\left(1+\left|\xi^{2}\right|\right)^{j} d \xi
$$

where $\xi=\left(\xi_{1}, \ldots, \xi_{n}\right)$ is the Fourier transform variable, and (2.2) applies for all real $j$.

In the following, $X_{h}^{(k)}$ and $Y_{h}^{(k-2 s)}$ are one-parameter families of Hilbert spaces; $X_{h}^{(k)}$ denotes the trial space, assumed of order $k$, and $Y_{h}^{(k-2 s)}$ denotes the test space, assumed of order $k-2 s$, where $s$ is an integer or possibly half integer, and the parameter $h$ is the mesh size. For all $j<k$ such that $X_{h}^{(k)} \subset H^{j}, g \in H^{k}$, we assume

$$
\underset{g_{x} \in X_{h}^{(k)}}{\operatorname{Inf}}\left\|g-g_{x}\right\|_{j} \leqslant C h^{k-j}\|g\|_{k}
$$

and for all $j<k-2 s$ such that $Y_{h}^{(k-2 s)} \subset H^{j}, g \in H^{k-2 s}$,

$$
\operatorname{Inf}_{g_{y} \in Y_{h}^{(k-2 s)}}\left\|g-g_{y}\right\|_{j} \leqslant C h^{k-2 s-j}\|g\|_{k-2 s} .
$$

We also assume that there exists a one-parameter $(h)$ family of symmetric pseudo-differential operators, denoted by $A_{h}$, with symbol $a(h \xi)$, with the property that $A_{h}^{2}$ maps 
$Y_{h}^{(k-2 s)}$ into $X_{h}^{(k)}$. The Galerkin procedure is included as a special case, with $A_{h} \equiv I$, $s=0, X_{h}^{(k)}=Y_{h}^{(k)}$.

Our approximation to the solution of $(2.1)$ is denoted by $u_{h} \in X_{h}^{(k)}$ which satisfies

$$
\left(L u-L u_{h}, y\right)=0 \quad \text { for all } y \in Y_{h}^{(k-2 s)} .
$$

Set $r=k-s-m ; O\left(h^{2 r}\right)$ is the optimal rate of convergence that can be expected for the method (2.3) [2] , [25]. We have the following result:

THEOREM 1. Suppose

(1) $X_{h}^{(k)}$ and $Y_{h}^{(k-2 s)}$ are subspaces of $H^{m}$ (we will assume that this implies $r>s)$;

(2) $A_{h}^{2}$ maps $Y_{h}^{(k-2 s)}$ onto $X_{h}^{(k)}$;

(3) $\left\|A_{h}\right\|_{0}=\sup _{\phi \in H^{0}}\left\|A_{h} \phi\right\|_{0} /\|\phi\|_{0}$ is bounded independently of $h$;

(4) the symbol $a(\cdot)$ is Hölder continuous in $h \xi$, with exponent $\gamma \in(0,1]$;

(5) $A_{h}$ is inverse bounded on $Y_{h}^{(k-2 s)}$, i. e. for all $y \in Y_{h}^{(k-2 s)},\|y\|_{m} \leqslant$ $C\left\|A_{h} y\right\|_{m}$, with $C$ independent of $h$;

then for sufficiently small $h$, there exists a unique solution to (2.3), and for all real $j \leqslant m$,

$$
\left\|u-u_{h}\right\|_{j} \leqslant C\left(h^{k-j}+h^{2 r}\right)\|u\|_{k} .
$$

Before proving the theorem, we comment on some implications of the hypotheses. We need test functions in $H^{m}$, so that collocation schemes are not covered. In fact, we could not expect (2.4) for collocation schemes, because $u \in H^{k}$ does not imply $L u$ continuous in sufficiently many dimensions. Hypotheses (2), (3), and (5) together imply the equality of dimension of the trial and test spaces. Hypothesis (5) may be viewed as a form of an inverse assumption on the space $X_{h}^{(k)}$, and for lack of a better name we shall refer to it as the "strong inverse assumption" below.

The proof of Theorem 1 requires a lemma, which is also in [29].

LEMMA 1. Let $M$ be a partial differential operator of order $p$ with smooth coefficients, and let $\phi \in H^{j}$ and $\psi \in H^{p-j}$. Assume hypothesis (4) holds, then

$$
\left|\left(\left(M A_{h}-A_{h} M\right) \phi, \psi\right)\right| \leqslant C h^{\gamma}\|\phi\|_{j}\|\psi\|_{p-j}, \quad 0 \leqslant j \leqslant p .
$$

Proof. A typical term of $M$ is of the form $b_{\alpha}(x) D^{\alpha},|\alpha| \leqslant p$, with the usual multi-index notation. The contribution of such a term to the left side of $(2.5)$ is given by

$$
\begin{aligned}
& \left|\iint d \xi d \xi^{\prime} \hat{\psi}(\xi) \hat{b}_{\alpha}\left(\xi^{\prime}-\xi\right)\left(a\left(h \xi^{\prime}\right)-a(h \xi)\right)\left(\xi^{\prime}\right)^{\alpha} \hat{\phi}\left(\xi^{\prime}\right)\right| \\
& \leqslant C h^{\gamma} \iint d \xi d \xi^{\prime}|\hat{\psi}(\xi)|\left|\hat{b}_{\alpha}\left(\xi^{\prime}-\xi\right)\right|\left|\xi^{\prime}-\xi\right|^{\gamma}\left[1+\left|\xi^{\prime}\right|^{j}\left(|\xi|^{p-j}+\left|\xi^{\prime}-\xi\right|^{p-j}\right)\right]\left|\hat{\phi}\left(\xi^{\prime}\right)\right| \\
& \quad \leqslant C h^{\gamma} \frac{\|\phi\|_{j}}{\|\psi\|_{p-j}} \iint d \xi d \xi^{\prime}|\hat{\psi}(\xi)|^{2}\left|b_{\alpha}\left(\xi^{\prime}-\xi\right)\right|\left|\xi^{\prime}-\xi\right|^{\gamma} \\
& \quad \cdot\left[1+|\xi|^{2(p-j)}+\left|\xi^{\prime}-\xi\right|^{2(p-j)}\right] \\
& \quad+C h^{\gamma} \frac{\|\psi\|_{p-j}}{\|\phi\|_{j}} \iint d \xi d \xi^{\prime}\left|b_{\alpha}\left(\xi^{\prime}-\xi\right)\right|\left|\xi^{\prime}-\xi\right|^{\gamma}\left[1+\left|\xi^{\prime}\right|^{2 j}\right]\left|\hat{\phi}\left(\xi^{\prime}\right)\right|^{2}
\end{aligned}
$$




$$
\begin{aligned}
& \leqslant C h^{\gamma} \frac{\|\phi\|_{j}}{\|\psi\|_{p-j}} \iint d \xi d \xi^{\prime}|\hat{\psi}(\xi)|^{2}\left[1+|\xi|^{2(p-j)}\right]\left|b_{\alpha}\left(\xi^{\prime}\right)\right|\left|\xi^{\prime}\right|^{\gamma}\left[1+\left|\xi^{\prime}\right|\right]^{2(p-j)} \\
& \quad+C h^{\gamma} \frac{\|\psi\|_{p-j}}{\|\phi\|_{j}} \iint d \xi d \xi^{\prime}\left|\hat{\phi}\left(\xi^{\prime}\right)\right|^{2}\left[1+\left|\xi^{\prime}\right|^{2 j}\right]\left|b_{\alpha}(\xi)\right||\xi|^{\gamma} \\
& \leqslant C h^{\gamma}\|\psi\|_{p-j}\|\phi\|_{j} .
\end{aligned}
$$

Proof of Theorem 1. Let $u_{x}$ be some optimum approximation in $X_{h}^{(k)}$ to $u$, and choose $y$ in (2.3) so that $A_{h}^{2} y=u_{x}-u_{h}$, using hypothesis (2). Then (2.3) becomes

$$
\begin{aligned}
0 & =\left(L u-L u_{h}, y\right)=\left(L u-L u_{x}, y\right)+\left(L A_{h}^{2} y, y\right) \\
& =\left(L\left(u-u_{x}\right), y\right)+\left(\left(L A_{h}-A_{h} L\right) A_{h} y, y\right)+\left(L A_{h} y, A_{h} y\right),
\end{aligned}
$$

using the symmetric property of $A_{h}$. We use the positive definiteness of $L$ and apply Lemma 1 with $M=L, p=2 m, j=m$, to obtain

$$
\begin{aligned}
\left\|A_{h} y\right\|_{m}^{2} & \leqslant C h^{\gamma}\|y\|_{m}\left\|A_{h} y\right\|_{m}+C\left\|u-u_{x}\right\|_{m}\|y\|_{m} \\
& \leqslant C h^{\gamma}\left\|A_{h} y\right\|_{m}^{2}+C\left\|u-u_{x}\right\|_{m}\left\|A_{h} y\right\|_{m}
\end{aligned}
$$

using the strong inverse assumption, hypothesis (5). For sufficiently small $h$, the existence and uniqueness of $y$ (and thus of $u_{h}$ ) is apparent from (2.7), by an application of the Lax-Milgram theorem, and we have

$$
\begin{aligned}
\left\|u-u_{h}\right\|_{m} & \leqslant\left\|u-u_{x}\right\|_{m}+\left\|A_{h}^{2} y\right\|_{m} \leqslant\left\|u-u_{x}\right\|_{m}+\left\|A_{h} y\right\|_{m} \\
& \leqslant C\left\|u-u_{x}\right\|_{m} \leqslant C h^{r+s}\|u\|_{k}
\end{aligned}
$$

using the boundedness of $A_{h}$, hypothesis (3), and the approximation property of $X_{h}^{(k)}$.

A simplified form of the Nitsche trick [21], [25, p. 166] gives the result for lower norms,

$$
\begin{aligned}
\left\|u-u_{h}\right\|_{k-2 r}^{2} & =\left(u-u_{h},(1-\Delta)^{k-2 r}\left(u-u_{h}\right)\right) \\
& =\left(L\left(u-u_{h}\right), L^{-*}(1-\Delta)^{k-2 r}\left(u-u_{h}\right)\right) \\
& =\left(L\left(u-u_{h}\right),\left(I-P_{0}\right) L^{-*}(1-\Delta)^{k-2 r}\left(u-u_{h}\right)\right) \\
& \leqslant C\left\|u-u_{h}\right\|_{m}\left\|\left(I-P_{0}\right) L^{-*}(1-\Delta)^{k-2 r}\left(u-u_{h}\right)\right\|_{m} \\
& \leqslant C h^{r+s}\|u\|_{k} h^{k-2 s-m}\left\|L^{-*}(1-\Delta)^{k-2 r}\left(u-u_{h}\right)\right\|_{k-2 s} \\
& \leqslant C h^{2 r}\|u\|_{k}\left\|u-u_{h}\right\|_{k-2 r},
\end{aligned}
$$

where $L^{-*}$ is the formal adjoint of $L^{-1}$ and $P_{0}$ is the projection into $Y_{h}^{(k-2 s)}$ with respect to the $H^{m}$ inner product.

Corollary. Suppose $X_{h}^{(k)} \subset H^{m+2 s}$ and $A_{h}$ is elliptic of order-s, i.e.

Then in (2.4), $j$ may be taken up to $m+2 s$.

Proof. Combining (2.7), (2.8) we obtain

$$
\begin{aligned}
\left\|u-u_{h}\right\|_{m+2 s} & \leqslant\left\|u-u_{x}\right\|_{m+2 s}+\left\|A_{h}\left(A_{h} y\right)\right\|_{m+2 s} \\
& \leqslant C h^{r-s}\|u\|_{k}+C h^{-2 s}\|y\|_{m} \\
& \leqslant C h^{r-s}\|u\|_{k}+C h^{-2 s}\left\|A_{h} y\right\|_{m} \\
& \leqslant C h^{r-s}\|u\|_{k} .
\end{aligned}
$$


If $u_{h}$ has any finite higher norms, estimates in these may be obtained by an inverse assumption in the usual way.

Estimates are also obtained for parabolic problems, using the above results and the general technique of Douglas and Dupont [6], [14]. We consider the problem

$$
\partial v / \partial t+L v=f, \quad(x, t) \in R^{n} \times(0, T), \quad v(x, 0)=g(x),
$$

where $L$ is as above. For simplicity, we consider the continuous time approximation and assume that the coefficients in $L$ do not depend on $t$. At each $t$, our approximation $v_{h}(t) \in X_{h}^{(k)}$ and satisfies

$$
\left(\partial v_{h} / \partial t+L v_{h}-f, y\right)=0 \text { for all } y \in Y_{h}^{(k-2 s)} .
$$

We choose $v_{h}(0)=P_{1} g$, where $P_{1}$ is a projection into $X_{h}^{(k)}$ induced by $L$, i.e.

$$
(L \phi, y)=\left(L P_{1} \phi, y\right) \text { for all } y \in Y_{h}^{(k-2 s)} .
$$

THEOREM 2. Suppose hypotheses (1)-(5) hold and $h$ is sufficiently small; then for all $T \geqslant 0$,

$$
\left\|v(T)-v_{h}(T)\right\|_{0} \leqslant C\left(h^{k}+h^{2 r}\right)\left[\|v(T)\|_{k}+\left(\int_{0}^{T}\left\|\frac{\partial v(t)}{\partial t}\right\|_{k-m}^{2} d t\right)^{1 / 2}\right] .
$$

Proof. In (2.10), choose $y$ such that $A_{h}^{2} y=v_{h}-P_{1} v$. Subtracting (2.9) and using (2.11), we obtain

$$
\left(A_{h}^{2} \frac{\partial y}{\partial t}+L A_{h}^{2} y, y\right)=\left(\frac{\partial}{\partial t}\left(v-P_{1} v\right), y\right)
$$

and since $A_{h}$ commutes with time differentiation, we have

$$
\begin{aligned}
\left(A_{h} \frac{\partial y}{\partial t}, A_{h} y\right) & =\left(\left(A_{h} L-L A_{h}\right) A_{h} y, y\right)-\left(L A_{h} y, A_{h} y\right)+\left(\left(I-P_{1}\right) \frac{\partial v}{\partial t}, y\right) \\
& \leqslant C h^{\gamma}\left\|A_{h} y\right\|_{m}\|y\|_{m}-C\left\|A_{h} y\right\|_{m}^{2}+\left\|\left(I-P_{1}\right) \frac{\partial v}{\partial t}\right\|_{-m}\|y\|_{m} \\
& \leqslant C h^{\gamma}\left\|A_{h} y\right\|_{m}^{2}-C\left\|A_{h} y\right\|_{m}^{2}+C\left(h^{k}+h^{2 r}\right)\left\|\frac{\partial v}{\partial t}\right\|_{k-m}\left\|A_{h} y\right\|_{m} \\
& \leqslant C\left(h^{2 k}+h^{4 r}\right)\left\|\frac{\partial v}{\partial t}\right\|_{k-m}^{2}
\end{aligned}
$$

using Lemma 1 for the commutator term, the regularity theory of [14] and Theorem 1 to estimate the last term. Thus

$$
\frac{d}{d t}\left\|A_{h} y\right\|_{0}^{2} \leqslant C\left(h^{2 k}+h^{4 r}\right)\left\|\frac{\partial v}{\partial t}\right\|_{k-m}^{2}
$$

and the initial condition was selected so that $\left\|A_{h} y(0)\right\|_{0}=0$. The proof is completed by a second application of Theorem 1 , to obtain

$$
\left\|v(t)-P_{1} v(t)\right\|_{0} \leqslant C\left(h^{k}+h^{2 r}\right)\|v(t)\|_{k}
$$

and using the boundedness of $A_{h}$, the triangle inequality, and the definition of $y$.

In Theorems 1,2 , the requirement of $H^{m}$ test functions can be relaxed if a stronger assumption is made about the smoothing properties of $A_{h}$.

The following theorem is given for the case $m=1$ for simplicity; a generalization to larger $m$ is not difficult. 
THEOREM 3. Suppose $m=1$, hypotheses (2), (3), (5) hold, and hypothesis (4) is replaced by the stronger requirement

(4') For each positive integer $j \leqslant n$, a Lipschitz condition of the form

$$
\left|\xi_{j} a\left(h \xi^{\prime}\right)-\xi_{j}^{\prime} a\left(h \xi^{\prime}\right)\right| \leqslant C\left|\xi-\xi^{\prime}\right|
$$

holds for all $\xi, \xi^{\prime} \in R^{n}$, with $C$ independent of $h$.

Then the conclusions of Theorems 1, 2 remain valid if hypothesis (1) is replaced by the weaker requirement

$$
Y_{h}^{(k-2 s)} \subset H^{0}, A_{h} Y_{h}^{(k-2 s)} \subset H^{1} .
$$

Proof. It suffices to obtain a sharper estimate for the commutator term in (2.6), (2.13), specifically to show that

$$
\left|\left(\left(L A_{h}-A_{h} L\right) A_{h} y, y\right)\right| \leqslant C\left\|A_{h} y\right\|_{1}\|y\|_{0}
$$

for all $y \in Y_{h}^{(k-2 s)}$. A typical term in $L$ is of the form $b(x)\left(\partial / \partial x_{i}\right)^{2}$, and the contribution of such a term to the left side of (2.14) is of the form

$$
\left|\iint d \xi d \xi^{\prime} \hat{y}\left(\xi^{\prime}\right)\left(a(h \xi)-a\left(h \xi^{\prime}\right)\right) \hat{b}\left(\xi^{\prime}-\xi\right) \xi_{i}^{2} a(h \xi) \hat{y}(\xi)\right|
$$

in (2.15), we use the estimate

$$
\left|\xi_{i}\left(a(h \xi)-a\left(h \xi^{\prime}\right)\right)\right| \leqslant\left|\xi_{i} a(h \xi)-\xi_{i}^{\prime} a\left(h \xi^{\prime}\right)\right|+\left|\xi_{i}-\xi_{i}^{\prime}\right|\left|a\left(h \xi^{\prime}\right)\right| \leqslant C\left|\xi-\xi^{\prime}\right|
$$

using hypotheses (3), (4'). Inserting (2.16) and estimating the expression (2.15) as in the proof of Lemma 1, (2.14) follows.

3. Tensor Products of Smooth Splines. Let $S_{h}^{(j)}$ denote the space of smooth $n$-dimensional (tensor product) splines of order $j$, i. e. piecewise polynomials of degree $j-1$, elements of $C^{j-2}$, on a uniform mesh of size $h$.

THEOREM 4. Suppose $X_{h}^{(k)} \equiv S_{h}^{(k)}, Y_{h}^{(k-2 s)} \equiv S_{h}^{(k-2 s)}$, s is a nonnegative integer, and $k \geqslant m+2 s+1$, then hypotheses (1)-(5), (4') are satisfied and Theorems 1-3 hold.

Proof. The approximation properties of these spaces are well known [1], and will not be discussed. Hypothesis (1) is immediate from the conditions on $s$ and $k$. We can choose $A_{h}^{2}$ to map $B$-splines in $S_{h}^{(k-2 s)}$ into $B$-splines in $S_{h}^{(k)}$, in which case hypothesis (2) is apparent and the symbol $a(\cdot)$ is given by

$$
a(h \xi)=\prod_{i=1}^{n} \frac{\sin ^{s}\left(h \xi_{i} / 2\right)}{\left(h \xi_{i} / 2\right)^{s}} .
$$

Hypotheses (3), (4), (4'), and the ellipticity condition (2.8) are apparent from (3.1). Thus, it is only the strong inverse assumption which requires proof; and each dimension can be treated independently, as $A_{h}$ is a product of one-dimensional mappings.

The theorem follows from the following lemma and the ordinary inverse assumption for splines.

LEMMA 2. Suppose $j, p$ are nonnegative integers with $0 \leqslant j \leqslant p-2, \phi$ is $a$ (onedimensional) spline of order $p$, and $A$ is a pseudo-differential operator with symbol $\sin (h \xi / 2) /(h \xi / 2)$. Then $A^{-1} \phi$ is defined and 


$$
\left|A^{-1} \phi\right|_{j} \leqslant|\phi|_{j}+C h|\phi|_{j+1},
$$

where $|\cdot|_{j}$ denotes the $H^{j}$-seminorm.

Proof. We set $z=h \xi$; noting that $\sin ^{2}(z / 2)=1 / 2(1-\cos z)$, we have

$$
\begin{aligned}
|\phi|_{j}^{2} & =4^{p} h^{-2 j-1} \int_{-\infty}^{\infty} q(z) z^{-2(p-j)} \sin ^{2}\left(\frac{z}{2}\right) d z, \\
\left|A^{-1} \phi\right|_{j}^{2} & =4^{p-1} h^{-2 j-1} \int_{-\infty}^{\infty} q(z) z^{-2(p-j-1)} d z,
\end{aligned}
$$

in which $q(z)$ is a nonnegative definite trigonometric polynomial in $z$, with a sufficiently high order zero at $z=0$ that the integrands are defined there. We may take $q(z)$ to be a finite polynomial, in which case both integrands are entire functions of $z$, and the integrals may be evaluated by the standard method of residues. Let $D \equiv d / d z$ and $N$ be a linear operator on trigonometric polynomials such that

$$
N e^{i \alpha z}= \begin{cases}e^{i \alpha z}, & \alpha \geqslant 0, \\ 0, & \alpha<0,\end{cases}
$$

then we have from (3.3)

$$
2 \pi i|\phi|_{j}^{2}=\left.\frac{4^{p} h^{-2 j-1}}{[2(p-j)-1] !}\left[D^{2(p-j)-1} N\left(q \sin ^{2}\left(\frac{z}{2}\right)\right)\right]\right|_{z=0} .
$$

Because $q$ is a trigonometric polynomial in $z$ and $2(p-j)-1 \geqslant 3$, one factor of $\sin (z / 2)$ can be placed to the left of $N$; then at least one of the derivatives must go on this factor, so that

$$
\begin{aligned}
2 \pi i|\phi|_{j}^{2}= & \left.\frac{4^{p} h^{-2 j-1}}{[2(p-j)-1] !}\left[D^{2(p-j)-1} \sin \left(\frac{z}{2}\right) N\left(q \sin \left(\frac{z}{2}\right)\right)\right]\right|_{z=0} \\
= & \left.\frac{1}{2} \frac{4^{p} h^{-2 j-1}}{[2(p-j)-2] !}\left[D^{2(p-j)-2} \cos \left(\frac{z}{2}\right) N\left(q \sin \left(\frac{z}{2}\right)\right)\right]\right|_{z=0} \\
& +\left.h^{-2 j-1} \sum_{\alpha=1,3, \ldots, 2(p-j)-3} C_{\alpha} D^{\alpha}\left[\sin \left(\frac{z}{2}\right) N\left(q \sin \left(\frac{z}{2}\right)\right)\right]\right|_{z=0},
\end{aligned}
$$

where the second term arises from the fact that any odd number of derivatives may be placed on the factor $\sin (z / 2)$ outside $N$. Since $2(p-j)-2$ is still strictly positive, we can put the cosine factor back inside $N$ and take the other sine factor out, obtaining

$$
\begin{aligned}
2 \pi i|\phi|_{j}^{2}= & \left.\frac{1}{2} \frac{4^{p} h^{-2 j-1}}{[2(p-j)-2] !}\left[D^{2(p-j)-2} N\left(q \sin \left(\frac{z}{2}\right) \cos \left(\frac{z}{2}\right)\right)\right]\right|_{z=0} \\
& +\left.h^{-2 j-1} \sum_{\alpha} C_{\alpha}\left[D^{\alpha} N\left(q \sin ^{2}\left(\frac{z}{2}\right)\right)\right]\right|_{z=0} \\
= & \left.\frac{1}{2} \frac{4^{p} h^{-2 j-1}}{[2(p-j)-2] !}\left[D^{2(p-j)-2} \sin \left(\frac{z}{2}\right) N\left(q \cos \left(\frac{z}{2}\right)\right)\right]\right|_{z=0}+\sum_{\alpha} .
\end{aligned}
$$

As before, one or any odd number of the remaining derivatives has to go on the sine factor, so this becomes 


$$
\begin{aligned}
2 \pi i|\phi|_{j}^{2}= & \left.\frac{4^{p-1} h^{-2 j-1}}{[2(p-j)-3] !}\left[D^{2(p-j)-3} \cos \left(\frac{z}{2}\right) N\left(q \cos \left(\frac{z}{2}\right)\right)\right]\right|_{z=0}+\sum_{\alpha} \\
& +\left.h^{-2 j-1} \sum_{\beta=1,3, \ldots, 2(p-j)-5} C_{\beta}^{\prime}\left[D^{\beta} \cos \left(\frac{z}{2}\right) N\left(q \cos \left(\frac{z}{2}\right)\right)\right]\right|_{z=0},
\end{aligned}
$$

where the $\Sigma_{\beta}$ term is understood to be simply dropped if $p-j=2$. Since $2(p-j)-3$ $\geqslant 1$ and $\beta \geqslant 1$ if the last sum is present, we can put the cosine factors back inside $N$, obtaining finally

$$
\begin{aligned}
2 \pi i|\phi|_{j}^{2}= & \left.\frac{4^{p-1} h^{-2 j-1}}{[2(p-j)-3] !}\left[D^{2(p-j)-3} N\left(q\left(1-\sin ^{2}\left(\frac{z}{2}\right)\right)\right)\right]\right|_{z=0} \\
& +\left.h^{-1-2 j} \sum_{\beta=1,3, \ldots, 2(p-j)-5} C_{\beta}^{\prime}\left[D^{\beta} N\left(q\left(1-\sin ^{\alpha}\left(\frac{z}{2}\right)\right)\right)\right]\right|_{z=0} .
\end{aligned}
$$

Using (3.3), (3.4), each term on the right side of (3.6) admits an interpretation as a seminorm of $\phi$ or $A^{-1} \phi$, in the same manner as (3.5) was obtained. We have

$$
\begin{aligned}
|\phi|_{j}^{2}= & \left|A^{-1} \phi\right|_{j}^{2}-C h^{2}|\phi|_{j+1}^{2}+\sum_{\alpha=1}^{p-j-1} C_{\alpha} h^{2 \alpha}|\phi|_{j+\alpha}^{2} \\
& +\sum_{\beta=1}^{p-j-2} C_{\beta}^{\prime} h^{2 \beta}\left(\left|A^{-1} \phi\right|_{j+\beta}^{2}-|\phi|_{j+\beta}^{2}\right),
\end{aligned}
$$

in which $\alpha, \beta, C_{\alpha}, C_{\beta}^{\prime}$ have been redefined; but the last sum is still omitted for $p=$ $j+2$. Since $p-j \geqslant 2$, each of the terms in (3.7) is well defined. We set $j=p-2$ initially, and let $j$ descend to its desired value in steps of one. Applying the usual inverse assumption at each step, the result (3.2) follows.

4. A Superconvergence Mechanism. Suppose $Z_{h}^{(k-s)}=A_{h} Y_{h}^{(k-2 s)}$ is also a space, of order $k-s$, as is the case for splines and some other spaces discussed in Section 6 . We assume hereafter that $s$ is positive, which is for our purposes the more interesting case. The results of Section 2 then suggest that for elliptic and parabolic problems, the accuracy of Galerkin's method in $Z_{h}^{(k-s)}$ can be improved by interpreting the approximate solution as an element of $X_{h}^{(k)}=A_{h} Z_{h}^{(k-s)}$ rather than as an element of $Z_{h}^{(k-s)}$. In this section we obtain conditions under which this is so. An alternative procedure for improving the order of accuracy by local averaging is described in [3] .

We consider the elliptic case first. Let $u_{z} \in Z_{h}^{(k-s)}$ be the Galerkin approximation to the solution of (2.1),

$$
\left(L u-L u_{z}, \psi\right)=0 \quad \text { for all } \psi \in Z_{h}^{(k-s)},
$$

then it is well known [24] that

$$
\left\|u-u_{z}\right\|_{j} \leqslant C\left(h^{k-s-j}+h^{2 r}\right)\|u\|_{k-s} .
$$

THEOREM 5. Let $u_{z}$ be as above, suppose hypotheses (1)-(3) hold, hypothesis (4) holds with $\gamma=1(a(\cdot)$ Lipschitz), and hypothesis (5) holds in the stronger form

$$
\|\phi\|_{j} \leqslant C\left\|A_{h} \phi\right\|_{j}, \quad \phi \in Y_{h}^{(k-2 s)} \text { or } \phi \in Z_{h}^{(k-s)}, \quad j=0,1, \ldots, m \text {. }
$$


Suppose $A_{h}$ is elliptic of order -s, i.e. (2.8) holds, and in addition

(6) $a(h \xi) \leqslant 1+O\left((h|\xi|)^{p}\right), p=\operatorname{maximum}(2 r, r+2 s-m-2)$;

(7) $L=L_{1}+L_{2}$, where $L_{1}$ has constant coefficients and $L_{2}$ is of order $2 m+1-s ;$ then

$$
\left\|u-A_{h} u_{z}\right\|_{j} \leqslant C\left(h^{k-j}+h^{2 r}\right)\|u\|_{k}, \quad j \leqslant m+2 s .
$$

Several comments are made before proving this theorem.

The form (4.3) of the strong inverse assumption is implied by Lemma 2. Hypothesis (6) can presumably be achieved by a change of basis in $X_{h}^{(k)}$, for smooth splines this is done in [28]; such a change of basis must be stable, however, so that (4.3) is preserved. The hypothesis (6) is viewed simply as a practical requirement for the construction of a suitable $A_{h}$.

The hypothesis (7) is no restriction for $s=1$; for larger $s$, it requires that some leading terms in $L$ have constant coefficients. In this respect and in the sense that $A_{h}$ defines a local mapping, our hypotheses resemble those of [8]. (Stronger results have been obtained for one-dimensional problems, however, in [9].) Our results are valid in an arbitrary number of dimensions, which is not the case for many superconvergence theorems; we note [11], however, as an interesting two-dimensional result, and [26], [27].

For $j>m+2 s$ in (4.5), the usual comments about inverse assumptions apply.

Proof of Theorem 5. As in Section 2, let $u_{x} \in X_{h}^{(k)}$ be an optimal approximation to $u$, let $y \in Y_{h}^{(k-2 s)}$, and set $\psi=A_{h} y$ in (4.1); we obtain

$$
\left(L u-L A_{h} u_{z}, y\right)=\left(L\left(I-A_{h}\right) u, y\right)+\left(\left(L A_{h}-A_{h} L\right)\left(u-u_{z}\right), y\right) .
$$

This is almost the same discrete equation as satisfied by $u_{h}$, Eq. (2.3); we may identify the first right-hand term of (4.8) as due to the different treatment of the inhomogeneous term, and the second term as due to variable coefficients in $L$. In the special case where $L$ has constant coefficients, this second term vanishes, and we observe the equivalence of the discrete equations.

Proceeding in the same way as to obtain (2.6), we choose $y$ in (4.6) such that $A_{h}^{2} y=u_{x}-A_{h} u_{z}$, then

$$
\begin{aligned}
\left(L A_{h} y, A_{h} y\right)= & -\left(L\left(u-u_{x}\right), y\right)+\left(L\left(I-A_{h}\right) u, y\right) \\
& +\left(\left(L A_{h}-A_{h} L\right)\left(u-u_{z}\right), y\right)-\left(\left(L A_{h}-A_{h} L\right) A_{h} y, y\right) \\
= & -\left(L\left(u-u_{x}\right), y\right)+\left(L\left(I-A_{h}\right) u, y\right)+\left(\left(L A_{h}-A_{h} L\right)\left(u-A_{h}^{-1} u_{x}\right), y\right)
\end{aligned}
$$

in the last term we note that $L_{1}$ commutes with $A_{h}$, and we apply Lemma 1 with $M=L_{2}, p=2 m+1-s, j=p-m$, obtaining

$$
\begin{aligned}
\left\|A_{h} y\right\|_{m}^{2} & \leqslant C\left\|u-u_{x}\right\|_{m}\|y\|_{m}+C\left\|\left(I-A_{h}\right) u\right\|_{m}\|y\|_{m}+C h\left\|u-A_{h}^{-1} u_{x}\right\|_{m+1-s}\|y\|_{m} \\
& \leqslant C\|y\|_{m}\left[h^{k-m}\|u\|_{k}+h\left\|u-A_{h}^{-1} u_{x}\right\|_{m+1-s}\right] \\
& \leqslant C\left\|A_{h} y\right\|_{m}\left[h^{k-m}\|u\|_{k}+h\left\|u-A_{h}^{-1} u_{x}\right\|_{m+1-s}\right] .
\end{aligned}
$$

Now let $\phi_{z} \in Z_{h}^{(k-s)}$ be some optimal approximation to $u$, and $y_{1} \in Y_{h}^{(k-2 s)}$ be such that 


$$
A_{h} y_{1}=\phi_{z}-A_{h}^{-1} u_{x}
$$

then for $j \leqslant m$,

$$
\begin{aligned}
\left\|u-A_{h}^{-1} u_{x}\right\|_{j}^{2}= & \left((1-\Delta)^{j}\left(u-A_{h}^{-1} u_{x}\right), u-A_{h}^{-1} u_{x}\right) \\
= & \left((1-\Delta)^{j}\left(u-A_{h}^{-1} u_{x}\right), u-\phi_{z}+A_{h} y_{1}\right) \\
\leqslant & \left\|u-A_{h}^{-1} u_{x}\right\|_{j}\left\|u-\phi_{z}\right\|_{j}+\left((1-\Delta)^{j}\left(u-u_{x}\right), y_{1}\right) \\
& -\left((1-\Delta)^{j}\left(I-A_{h}\right) u, y_{1}\right),
\end{aligned}
$$

since $A_{h}$ commutes with $1-\Delta$. First suppose $j \geqslant 0$, then we estimate (4.10) using

$$
\left\|u-A_{h}^{-1} u_{x}\right\|_{j}^{2} \leqslant C h^{k-s-j}\|u\|_{k-s}\left[\left\|u-A_{h}^{-1} u_{x}\right\|_{j}+\left\|y_{1}\right\|_{j}\right]
$$

from (4.3) and (4.9),

$$
\begin{aligned}
\left\|y_{1}\right\|_{j} & \leqslant C\left\|A_{h} y_{1}\right\|_{j} \leqslant C\left\|u-\phi_{z}\right\|_{j}+C\left\|u-A_{h}^{-1} u_{x}\right\|_{j} \\
& \leqslant C h^{k-s-j}\|u\|_{k-s}+C\left\|u-A_{h}^{-1} u_{x}\right\|_{j} ;
\end{aligned}
$$

combining (4.11) and (4.12) gives

$$
\left\|u-A_{h}^{-1} u_{x}\right\|_{j} \leqslant C h^{k-s-j}\|u\|_{k-s} .
$$

For negative $j$ in (4.10), we proceed differently, obtaining

$$
\begin{aligned}
\left\|u-A_{h}^{-1} u_{x}\right\|_{j}^{2} \leqslant & C h^{k-s-j}\left\|u-A_{h}^{-1} u_{x}\right\|_{j}\|u\|_{k-s} \\
& +C\left\|y_{1}\right\|_{0}\left[\left\|u-u_{x}\right\|_{2 j}+\left\|\left(I-A_{h}\right) u\right\|_{2 j}\right]
\end{aligned}
$$

in (4.14) we use (4.12) and (4.13) with $j=0$ to estimate $\left\|y_{1}\right\|_{0}$, and (4.4) to estimate the last term, obtaining

$$
\begin{aligned}
\left\|u-A_{h}^{-1} u_{x}\right\|_{j}^{2} \leqslant C h^{k-s-j}\left\|u-A_{h}^{-1} u_{x}\right\|_{j}\|u\|_{k-s} & \\
& +C\left(h^{2(k-s-j)}+h^{2(k-m-1)}\right)\|u\|_{k-s}^{2}
\end{aligned}
$$

from which it follows that we may set $j=m+1-s$ in (4.13), regardless of the sign of $j$. Comparing this result with (4.8) then establishes (4.5) for $j=m$. As before,

$$
\begin{aligned}
& \left\|u-A_{h} u_{z}\right\|_{m+2 s} \leqslant\left\|u-u_{x}\right\|_{m+2 s}+\left\|u_{x}-A_{h} u_{z}\right\|_{m+2 s} \\
& \quad \leqslant C h^{r-s}\|u\|_{k}+C h^{-2 s}\left\|A_{h}^{-2} u_{x}-A_{h}^{-1} u_{z}\right\|_{m} \leqslant C h^{r-s}\|u\|_{k}+C h^{-2 s}\left\|u_{x}-A_{h} u_{z}\right\|_{m} \\
& \quad \leqslant C h^{r-s}\|u\|_{k}+C h^{-2 s}\left(\left\|u-u_{x}\right\|_{m}+\left\|u-A_{h} u_{z}\right\|_{m}\right) \leqslant C h^{r-s}\|u\|_{k} .
\end{aligned}
$$

For lower norms, we again use the Nitsche trick; let $\chi=L^{-*}(1-\Delta)^{k-2 r}\left(u-A_{h} u_{z}\right)$, $\theta=L\left(I-A_{h}\right) u+\left(L A_{h}-A_{h} L\right)\left(u-u_{z}\right)$ and $P_{0}$ the projection operator into $Y_{h}^{(k-2 s)}$ with respect to the $H^{m}$ inner product as in Section 2. Then

$$
\begin{aligned}
\left\|u-A_{h} u_{z}\right\|_{k-2 r}^{2} & =\left(u-A_{h} u_{z},(1-\Delta)^{k-2 r}\left(u-A_{h} u_{z}\right)\right) \\
& =\left(L u-L A_{h} u_{z},\left(I-P_{0}\right) \chi\right)+\left(L u-L A_{h} u_{z}, P_{0} \chi\right) \\
& =\left(L u-L A_{h} u_{z},\left(I-P_{0}\right) \chi\right)+\left(\theta, P_{0} \chi\right),
\end{aligned}
$$

using (4.6) with $y=P_{0} \chi$. Since $(1-\Delta)^{m} P_{0}$ is symmetric,

$$
\left(\theta, P_{0} \chi\right)=\left((1-\Delta)^{-m} \theta,(1-\Delta)^{m} P_{0} \chi\right)=\left((1-\Delta)^{m} P_{0}(1-\Delta)^{-m} \theta, \chi\right) ;
$$

and (4.16) may be estimated 


$$
\begin{aligned}
&\left\|u-A_{h} u_{z}\right\|_{k-2 r}^{2} \leqslant C\left\|u-A_{h} u_{z}\right\|_{m}\left\|\left(I-P_{0}\right) \chi\right\|_{m} \\
& \quad+\left\|(1-\Delta)^{m} P_{0}(1-\Delta)^{-m} \theta\right\|_{2 s-k}\|\chi\|_{k-2 s} \\
& \leqslant C h^{k-m}\|u\|_{k} \cdot h^{k-2 s-m}\|\chi\|_{k-2 s}+\left\|P_{0}(1-\Delta)^{-m} \theta\right\|_{k-2 r}\|\chi\|_{k-2 s}
\end{aligned}
$$

From the definition of $\chi$, it follows that

$$
\|\chi\|_{k-2 s} \leqslant C\left\|u-A_{h} u_{z}\right\|_{k-2 r} .
$$

Now we note that $k-2 r=k-2 s-2(k-2 s-m)$, and so $P_{0}$ is stable in $\|\cdot\|_{k-2 r} ;$ thus,

$$
\begin{aligned}
&\left\|P_{0}(1-\Delta)^{-m} \theta\right\|_{k-2 r} \leqslant\left\|(1-\Delta)^{-m} \theta\right\|_{k-2 r}+C h^{r-s}\left\|(1-\Delta)^{-m} \theta\right\|_{m} \\
& \leqslant C\|\theta\|_{2 s-k}+C h^{r-s}\|\theta\|_{-m} \leqslant C\left\|\left(I-A_{h}\right) u\right\|_{k-2 r}+C h^{r-s}\left\|\left(I-A_{h}\right) u\right\|_{m} \\
&+C\left\|\left(L A_{h}-A_{h} L\right)\left(u-u_{z}\right)\right\|_{2 s-k}+C h^{r-s}\left\|\left(L A_{h}-A_{h} L\right)\left(u-u_{z}\right)\right\|_{-m} \\
& \leqslant C h^{2 r}\|u\|_{k}+C h\left\|u-u_{z}\right\|_{m+1-r}+C h^{r+1-s}\left\|u-u_{z}\right\|_{m+1-s},
\end{aligned}
$$

where we have used Lemma 1 twice in the last step, with $M=L_{2}, p=2 m+1-s$, $j=m+1-r$ and $m+1-s$, with $\psi=(1-\Delta)^{2 s-k}\left(L_{2} A_{h}-A_{h} L_{2}\right)\left(u-u_{z}\right)$ and $(1-\Delta)^{-m}\left(L_{2} A_{h}-A_{h} L_{2}\right)\left(u-u_{z}\right)$. Finally, we can estimate the right side of (4.19) by (4.2); combining the result obtained with (4.17) and (4.18) establishes (4.5) for $j=k-2 r$, and so proves Theorem 5 .

As expected, Theorem 5 leads easily to a similar theorem for parabolic problems. Let $v_{z} \in Z_{h}^{(k-s)}$ be the continuous time Galerkin approximation to the solution $v$ of (2.9), i.e.

$$
\left(\partial v_{z} / \partial t+L v_{z}-f, \psi\right)=0 \text { for all } \psi \text { in } Z_{h}^{(k-s)} .
$$

For simplicity, we again assume that the coefficients of $L$ do not depend on $t$, and we let $P_{2}$ denote the Galerkin projection operator into $Z_{h}^{(k-s)}$ induced by $L$,

$$
\left(L \phi-L P_{2} \phi, \psi\right)=0 \text { for all } \psi \in Z_{h}^{(k-s)}, \phi \in H^{m} .
$$

We choose as the initial condition

$$
v_{z}(0)=P_{2} g
$$

in which case the following holds:

THEOREM 6. Suppose the hypotheses of Theorem 5 hold, and $v_{z}$ is determined as above. Then for all $T \geqslant 0$,

$$
\left\|v(T)-A_{h} v_{z}(T)\right\|_{0} \leqslant C\left(h^{k}+h^{2 r}\right)\left[\|v(T)\|_{k}+\int_{0}^{T}\left\|\frac{\partial v(t)}{\partial t}\right\|_{k-m} d t\right] .
$$

Proof.

$$
\begin{aligned}
\left\|v(T)-A_{h} v_{z}(T)\right\|_{0} & \leqslant\left\|\left(I-A_{h} P_{2}\right) v(T)\right\|_{0}+\left\|A_{h}\left(P_{2} v(T)-v_{z}(T)\right)\right\|_{0} \\
& \leqslant C\left(h^{k}+h^{2 r}\right)\|v(T)\|_{k}+C\left\|P_{2} v(T)-v_{z}(T)\right\|_{0},
\end{aligned}
$$

using Theorem 5 and the boundedness of $A_{h}$. From (2.9), (4.20), (4.21) it follows that for any $\psi \in Z_{h}^{(k-s)}, t \geqslant 0$,

$$
\left(\frac{\partial}{\partial t}\left(v_{z}-P_{2} v\right)+L\left(v_{z}-P_{2} v\right), \psi\right)=\left(\left(I-P_{2}\right) \frac{\partial v}{\partial t}, \psi\right)
$$


choosing $\psi=v_{z}-P_{2} v$, we obtain

$$
\begin{aligned}
& \left\|v_{z}-P_{2} v\right\|_{0} \frac{d}{d t}\left\|v_{z}-P_{2} v\right\|_{0} \\
& \quad \leqslant-C\left\|v_{z}-P_{2} v\right\|_{m}^{2}+\left(A_{h}\left(I-P_{2}\right) \frac{\partial v}{\partial t}, A_{h}^{-1}\left(v_{z}-P_{2} v\right)\right) \\
& \quad \leqslant-C\left\|v_{z}-P_{2} v\right\|_{m}^{2}+\left(\left\|\left(I-A_{h}\right) \frac{\partial v}{\partial t}\right\|_{-m}+\left\|\left(I-A_{h} P_{2}\right) \frac{\partial v}{\partial t}\right\|_{-m}\right)\left\|A_{h}^{-1}\left(v_{z}-P_{2} v\right)\right\|_{m} \\
& \quad \leqslant C\left(h^{2 k}+h^{4 r}\right)\left\|\frac{\partial v}{\partial t}\right\|_{k-m}^{2}
\end{aligned}
$$

again using Theorem 5. Since $\left\|v_{z}(0)-P_{2} v(0)\right\|_{0}=0$ by (4.22), (4.23) follows.

Pointwise superconvergence results are also obtained from Theorems 5 and 6.

For the elliptic case, let $\Omega_{h}=\left\{x \in R^{n} \mid u_{z}(x)=A_{h} u_{z}(x)\right\} . \Omega_{h}$ may depend on $h$, but it is not empty, as $a(0)=1$. We apply the elementary estimates

$$
\|\cdot\|_{L_{\infty}\left(R^{n}\right)} \leqslant \begin{cases}C\|\cdot\|_{0}^{1 / 2}\|\cdot\|_{1}^{1 / 2}, & n=1, \\ C\|\cdot\|_{0}^{1 / 4}\|\cdot\|_{1}^{1 / 2}\|\cdot\|_{2}^{1 / 4}, & n=2, \\ \epsilon\|\cdot\|_{2}+C \epsilon^{-1 / 2}\|\cdot\|_{1}, & n=3,\end{cases}
$$

to the function $u-A_{h} u_{z}$; in general,

but

$$
\left\|u-u_{z}\right\|_{L_{\infty}\left(R^{n}\right)} \geqslant O\left(h^{k-s}\right)
$$

$$
\operatorname{Sup}_{x \in \Omega_{h}}\left|u(x)-u_{z}(x)\right| \leqslant C\|u\|_{k} \begin{cases}h^{k-1 / 2}, & n=1, \\ h^{k-1}, & n=2, \\ h^{k-4 / 3}, & n=3,\end{cases}
$$

so that for $s \geqslant 2$, pointwise superconvergence occurs in up to three dimensions. Similar results hold for the parabolic case.

5. First Order Hyperbolic Equations. It suffices for the present discussion to consider the scalar equation in one space dimension,

$$
\frac{\partial w}{\partial t}+b \frac{\partial w}{\partial x}=f, \quad(x, t) \in R \times(0, T), \quad w(\cdot, 0)=g
$$

the generalization of our results to symmetric hyperbolic systems in $n$ space dimensions and including a linear undifferentiated term is immediate. In (5.1), $b$ and $f$ are smooth functions of $(x, t)$ and $g$ is a smooth function of $x$. We obtain continuous time approximations to $w$ in $X_{h}^{(k)}$, a finite element space of order $k$, as above; the test space $Y_{h}$ is not required to satisfy any approximation properties. We let $A_{h}$ be a symmetric pseudo-differential operator as above, with symbol $a(h \xi)$. As above, we have in mind the case where $a(h \xi)$ is of the form (3.1) with positive integer $s$. The case $s=0, A=I$ can be included by replacing the ellipticity hypothesis (iv) below by an inverse assumption on the space $X_{h}^{(k)}$.

THEOREM 7. Let $w_{h} \in X_{h}^{(k)} \times[0, T]$ be determined from the relations 


$$
\begin{gathered}
\left(\frac{\partial w_{h}}{\partial t}+b \frac{\partial w_{h}}{\partial x}-f, y\right)=0 \text { for all } y \in Y_{h}, t \in(0, T) \\
w_{h}(\cdot, 0)=P_{3} g
\end{gathered}
$$

where $P_{3}$ is the $H^{1}$ projection into $X_{h}^{(k)}$. Suppose that

(i) $X_{h}^{(k)} \subset H^{1}, Y_{h} \subset H^{0}$;

(ii) $A_{h}^{2}$ maps $Y_{h}$ onto $X_{h}^{(k)}$;

(iii) $A_{h}$ is bounded in $H^{0}$;

(iv) $A_{h}$ is elliptic of order-1 ((2.8) holds with $\left.s=1\right)$;

(v) $a(\cdot)$ is Lipschitz continuous in $h \xi$;

(vi) $A_{h}$ is inverse bounded on $Y_{h}\left(\|\phi\|_{0} \leqslant C\left\|A_{h} \phi\right\|_{0}\right.$ for all $\left.\phi \in Y_{h}\right)$; then for $t \geqslant 0$,

$$
\begin{aligned}
& \left\|w(\cdot, t)-w_{h}(\cdot, t)\right\|_{0} \\
& \quad \leqslant C(t) h^{k-1}\left[\|w(\cdot, t)\|_{k-1}+\int_{0}^{t}\left[\|w(\cdot, t)\|_{k}+\|f(\cdot, t)\|_{k-1}\right] d t\right] .
\end{aligned}
$$

Proof. Subtracting (5.2) from (5.1) in the usual way and choosing $y$ such that $A_{h}^{2} y=P_{3} w-w_{h}$, we obtain

$$
\begin{aligned}
&\left.\left(\frac{\partial A_{h}^{2} y}{\partial t}+b \frac{\partial A_{h}^{2} y}{\partial x}+\left(\frac{\partial}{\partial t}+b \frac{\partial}{\partial x}\right)\left(w-P_{3} w\right), y\right)\right)=0 \\
&\left(A_{h} y, \frac{\partial A_{h} y}{\partial t}\right)=-\left(A_{h} y, b \frac{\partial A_{h} y}{\partial x}\right)-\left(y,\left(b \frac{\partial}{\partial x} A_{h}-A_{h} b \frac{\partial}{\partial x}\right) A_{h} y\right) \\
&-\left(y,\left(\frac{\partial}{\partial t}+b \frac{\partial}{\partial x}\right)\left(w-P_{3} w\right)\right) \\
& \leqslant \frac{1}{2}\left(A_{h} y,\left(\frac{\partial b}{\partial x}\right) A_{h} y\right)+C h\|y\|_{0}\left\|A_{h} y\right\|_{1} \\
&+C\|y\|_{0}\left[\left\|\left(I-P_{3}\right) w\right\|_{1}+\left\|\left(I-P_{3}\right) \frac{\partial w}{\partial t}\right\|_{0}\right]
\end{aligned}
$$

using Lemma 1 with $\gamma=1, M=b \partial / \partial x, p=1, \phi=A_{h} y, \psi=y$. From the ellipticity and inverse bound on $A_{h}$, we have

$$
\frac{d}{d t}\left\|A_{h} y\right\|_{0}^{2} \leqslant C\left\|A_{h} y\right\|_{0}^{2}+C\left\|A_{h} y\right\|_{0}\left[\left\|\left(I-P_{3}\right) w\right\|_{1}+\left\|\left(I-P_{3}\right) f\right\|_{0}\right] ;
$$

since (5.3) implies $\left\|A_{h} y(\cdot, 0)\right\|_{0}=0$, this implies

$$
\begin{aligned}
\left\|P_{3} w(\cdot, t)-w_{h}(\cdot, t)\right\|_{0} & =\left\|A_{h}^{2} y\right\|_{0} \leqslant C\left\|A_{h} y\right\|_{0} \\
& \leqslant C h^{k-1} \int_{0}^{t}\left[\|w(\cdot, t)\|_{k}+\|f(\cdot, t)\|_{k-1}\right] d t
\end{aligned}
$$

from which (5.4) easily follows.

This is the same order of accuracy as obtained by Lesaint [17] for the Galerkin approximation. Theorem 6 shows that under suitable conditions, the same order of accuracy can be achieved with simple, e.g. piecewise constant test functions, and correspondingly simpler discrete equations.

The Galerkin approximation, however, is special. The following theorem contains Dupont's result for splines [13] as a special case (also implied by [27]). 
THEOREM 8. Let $w_{x}$ be the Galerkin approximation in $X_{h}^{(k)} \times[0, T]$ to $w, o b$ tained from the relations

$$
\begin{gathered}
\left(\frac{\partial w_{x}}{\partial t}+b \frac{\partial w_{x}}{\partial x}-f, \psi\right)=0 \text { for all } y \in Y_{h}, t \in(0, T), \\
w_{x}(\cdot, 0)=A_{h}^{-1} P_{4} g
\end{gathered}
$$

where $P_{4}$ is the projection into $E_{h}^{(k+1)}=A_{h} X_{h}^{(k)}$ with respect to the $H^{1}$ inner product. Suppose that $E_{h}^{(k+1)}$ is a space of order up to $k+1$, i. e., for $0 \leqslant j<m \leqslant k+1$,

$$
\operatorname{Inf}_{\chi \in E_{h}^{(k+1)}}\|\phi-\chi\|_{j} \leqslant C h^{m-j}\|\phi\|_{m} .
$$

Let $F_{h}=A_{h}^{-1} X_{h}^{(k)}$; and suppose that

(i) $F_{h} \subset H^{0}, X_{h}^{(k)} \subset H^{1}$;

(ii) $A_{h}^{2}$ maps $F_{h}$ onto $E_{h}^{(k+1)}$;

(iii) $A_{h}$ is bounded in $H^{0}$;

(iv) $a(\cdot)$ is Lipschitz continuous in $h \xi$;

(v) $|a(h \xi)| \leqslant 1+O\left((h \xi)^{k}\right)$;

(vi) $A_{h}$ is inverse bounded on $F_{h}$ and on $X_{h}^{(k)}$, i.e. $\|\phi\|_{0} \leqslant C\left\|A_{h} \phi\right\|_{0}$ for all $\phi \in F_{h}$ and $\|\psi\|_{1} \leqslant C\left\|A_{h} \psi\right\|_{1}$ for all $\psi \in X_{h}^{(k)}$;

then for all $t \geqslant 0$,

$$
\begin{aligned}
& \left\|w(\cdot, t)-w_{x}(\cdot, t)\right\|_{0} \\
& \quad \leqslant C(t) h^{k}\left[\|w(\cdot, t)\|_{k}+\int_{0}^{t}\left[\|w(\cdot, t)\|_{k+1}+\|f(\cdot, t)\|_{k}\right] d t\right] .
\end{aligned}
$$

Remark. We have not assumed $A_{h}$ to be inverse bounded on $F_{h} \cup X_{h}^{(k)}$, which is not true in general. For the case $a(h \xi)$ given by (3.1) multiplied by a trigonometric polynomial, hypotheses (ii)-(vi) are satisfied for $s$ a nonnegative integer. The approximation property required of $E_{h}^{(k+1)}$ precludes the case $s=0$. For $X_{h}^{(k)}$ the space of smooth splines of order $k$ with $s=1, E_{h}^{(k+1)}$ and $F_{h}$ are the spaces of splines of order $k+1$ and $k-1$, respectively.

Proof. We subtract (5.1) from (5.5) and proceed essentially as before. The idea is that $A_{h} w_{x}$ satisfies similar discrete equations, with $F_{h}$ thought of as the test space, as obtained in Theorem 6 for $u_{h}$. For any $y \in F_{h}$, we obtain

$$
\left(\frac{\partial}{\partial t}\left(A_{h} w-A_{h} w_{x}\right)+b \frac{\partial}{\partial x}\left(A_{h} w-A_{h} w_{x}\right)+\left(A_{h} b \frac{\partial}{\partial x}-b \frac{\partial}{\partial x} A_{h}\right)\left(w-w_{x}\right), y\right)=0
$$

choosing $A_{h}^{2} y=P_{4} w-A_{h} w_{x}$, this becomes

$$
\begin{aligned}
\left(\left(\frac{\partial}{\partial t}+b \frac{\partial}{\partial x}\right) A_{h}^{2} y, y\right)+ & \left(\left(\frac{\partial}{\partial t}+b \frac{\partial}{\partial x}\right)\left(A_{h} w-P_{4} w\right), y\right) \\
& +\left(\left(A_{h} b \frac{\partial}{\partial x}-b \frac{\partial}{\partial x} A_{h}\right)\left(w-w_{x}\right), y\right)=0 \\
\left(\frac{\partial}{\partial t} A_{h} y, A_{h} y\right)+ & \left(b \frac{\partial}{\partial x} A_{h} y, A_{h} y\right)+\left(\left(\frac{\partial}{\partial t}+b \frac{\partial}{\partial x}\right)\left(A_{h} w-P_{4} w\right), y\right) \\
+ & \left(\left(A_{h} b \frac{\partial}{\partial x}-b \frac{\partial}{\partial x} A_{h}\right)\left(w-w_{x}\right), y\right) \\
& -\left(\left(A_{h} b \frac{\partial}{\partial x}-b \frac{\partial}{\partial x} A_{h}\right)\left(A_{h}^{-1} P_{4} w-w_{x}\right), y\right)=0
\end{aligned}
$$


The two commutator terms can be combined; this is the special property of the Galerkin approximation. Integrating by parts and using Lemma 1 as above, we obtain from (5.8),

$$
\begin{aligned}
\left(\frac{\partial}{\partial t} A_{h} y, A_{h} y\right)= & \frac{1}{2}\left(A_{h} y,\left(\frac{\partial b}{\partial x}\right) A_{h} y\right)-\left(\left(\frac{\partial}{\partial t}+b \frac{\partial}{\partial x}\right)\left(A_{h} w-P_{4} w\right), y\right) \\
& +\left(\left(A_{h} b \frac{\partial}{\partial x}-b \frac{\partial}{\partial x} A_{h}\right)\left(w-A_{h}^{-1} P_{4} w\right), y\right),
\end{aligned}
$$

$$
\begin{aligned}
& \frac{d}{d t}\left\|A_{h} y\right\|_{0}^{2} \leqslant C\left\|A_{h} y\right\|_{0}^{2} \\
& +C\|y\|_{0}\left[\left\|\left(I-A_{h}\right) \frac{\partial w}{\partial t}\right\|_{0}+\left\|\left(I-P_{4}\right) \frac{\partial w}{\partial t}\right\|_{0}+\left\|\left(I-A_{h}\right) w\right\|_{1}\right. \\
& \left.+\left\|\left(I-P_{4}\right) w\right\|_{1}+h\left\|\left(I-A_{h}^{-1} P_{4}\right) w\right\|_{1}\right] \\
& \leqslant C\left\|A_{h} y\right\|_{0}^{2}+C\left\|A_{h} y\right\|_{0}\left[h^{k}\|w\|_{k+1}+h^{k}\|f\|_{k}+h\left\|\left(I-A_{h}^{-1} P_{4}\right) w\right\|_{1}\right] .
\end{aligned}
$$

Let $w_{0}$ be an optimal approximation in $X_{h}^{(k)}$ to $w$, then

$$
\begin{aligned}
& \left\|w-A_{h}^{-1} P_{4} w\right\|_{1} \leqslant\left\|w-w_{0}\right\|_{1}+\left\|w_{0}-A_{h}^{-1} P_{4} w\right\|_{1} \\
& \quad \leqslant C h^{k-1}\|w\|_{k}+\left\|A_{h} w_{0}-P_{4} w\right\|_{1} \\
& \leqslant C h^{k-1}\|w\|_{k}+\left\|w-P_{4} w\right\|_{1}+\left\|w-A_{h} w_{0}\right\|_{1} \\
& \leqslant C h^{k-1}\|w\|_{k}+\left\|\left(I-A_{h}\right) w\right\|_{1}+\left\|A_{h}\left(w-w_{0}\right)\right\|_{1} \leqslant C h^{k-1}\|w\|_{k}
\end{aligned}
$$

combining (5.9) and (5.10) and using (5.6) gives

$$
\left\|A_{h} y\right\|_{0} \leqslant C(t) h^{k} \int_{0}^{t}\left[\|w(\cdot, t)\|_{k+1}+\|f(\cdot, t)\|_{k}\right] d t
$$

Finally,

$$
\left\|w(\cdot, t)-w_{h}(\cdot, t)\right\|_{0} \leqslant\left\|\left(I-A_{h}^{-1} P_{4}\right) w\right\|_{0}+\left\|A_{h}^{2} y\right\|_{0}
$$

an argument similar to that used to obtain (5.10) gives $\left\|\left(I-A_{h}^{-1} P_{4}\right) w\right\|_{0} \leqslant C h^{k}\|w\|_{k}$, and thus (5.7).

6. Discussion. There are spaces other than smooth splines to which our formalism applies. For example, we can take $A_{h}^{2}$ to be the inverse of a partial differential operator (cf. [12], [27], e.g. $a(h \xi)=\left(1+h^{2}\left|\xi^{2}\right|\right)^{-1 / 2}$; with such a choice for $A_{h}$, our hypothesis (5) is simply an inverse assumption on $X_{h}^{(k)}$. Analogous to [12] , [27] we would then choose $X_{h}^{(k)}$ a space of piecewise polynomials of order $k$, and $Y_{h}^{(k-2 s)}=$ $A_{h}^{-2} X_{h}^{(k)}$.

The results of [10], [22] correspond to $A_{h}^{2}$ a family of partial differential operators; many of our results can be directly extended to this case.

The choice of $a(\cdot)$ given by (3.1) is special if we wish to generate a hierarchy of spaces by successive application of $A_{h}$. This is the choice which minimizes the radius of support of the higher order basis elements in the following sense.

THEOREM 9. Let $X_{h}^{(j)}$ be a space of order $j$, spanned by the uniform translates of tensor products of basis element $(s) \phi_{j}(x / h)$, the support of which is contained in 
$\left[-\rho_{j}, \rho_{j}\right] \quad\left(\rho_{j}\right.$ is the smallest such number). Suppose that for all nonnegative integer $p, X_{h}^{(j+p)}=A_{h}^{p} X_{h}^{(j)}$ is a space of order $j+p$. Let $\rho_{j+p}$ denote the radius of support of the basis elements of $X_{h}^{(j+p)}$. Then for sufficiently large $p, \rho_{j+p+1} \geqslant \rho_{j+p}+1 / 2$, and $\rho_{j+p+1}=\rho_{j+p}+1 / 2$ if and only if $a(\cdot)$ is given by (3.1), with $s$ set equal to 1 .

Proof. In the $x$-variables, $A_{h}$ is simply a convolution operator with kernel $\stackrel{\gamma}{a}(x / h)$ (the inverse Fourier transform of $a(h \xi)$ ). In order that $A_{h}$ successively generate spaces of all orders, $a(h \xi)=0$ for all $h \xi=2 \pi j, j$ any nonzero integer [25]. Thus, for any nonzero integer $j$,

$$
\int \check{a}\left(\frac{x}{h}\right) \exp \left(2 \pi i j \frac{x}{h}\right) d x=0 .
$$

If $\stackrel{r}{a}(x / h)$ has its support within $[-1 / 2,1 / 2]$, then by comparison of its Fourier series with (6.1), it follows that $\stackrel{\Upsilon}{a}(x / h)$ is constant in $(-1 / 2,1 / 2)$, which corresponds to $a(h \xi)$ given by (3.1) (with $s=1$ ).

However, this choice of $A_{h}$ maps piecewise polynomials into piecewise polynomials, and can be applied to other spaces. For example, if we start with the Hermite cubics, we obtain successively the $C^{2}$ piecewise quartics, the $C^{3}$ piecewise quintics, etc. Our results hold for such spaces (the modification of Lemma 2 in such cases is straightforward, but tedious).

Finally we consider the rates of convergence of Galerkin methods for first order symmetric hyperbolic systems. In [13], Dupont showed that in $L^{2}, O\left(h^{3}\right)$ was obtained for Hermite cubics and $O\left(h^{4}\right)$ for cubic splines. Our Theorem 8 holds for the cubic splines, but not for the Hermite cubics; for $X_{h}^{(k)}$ the space of Hermite cubics, $F_{h}=A_{h}^{-1} X_{h}^{(k)}$ is not a subspace of $H^{0}$. A different choice of $A_{h}$ will not help, because the Fourier transforms of the two basis elements of the Hermite cubic space do not have a suitable common factor. Our results do imply the optimal order of accuracy for the higher spaces in the hierarchy beginning with the Hermite cubics, however, for example, $O\left(h^{6}\right)$ for the Galerkin procedure with the $C^{3}$ piecewise quintics.

Department of Mathematics

Rutgers University

New Brunswick, Jersey 08903

1. J. H. AHLBERG, E. N. NILSON \& J. L. WALSH, The Theory of Splines and Their Applications, Academic Press, New York, 1967. MR 39 \#684.

2. I. BABUSKA \& A. K. AZIZ, "Survey lectures on the mathematical foundations of the finite element method," The Mathematical Foundations of the Finite Element Method with Applications to Partial Differential Equations, A. K. Aziz (editor), Academic Press, New York, 1972.

3. J. H. BRAMBLE \& A. M. SCHATZ, "Higher order local accuracy by averaging in the finite element method," Mathematical Aspects of Finite Elements in Partial Differential Equation, C. deBoor (editor), Academic Press, New York, 1974.

4. C. deBOOR, The Method of Projections as Applied to the Numerical Solution of Two Point Boundary Value Problems Using Cubic Splines, Ph. D. Thesis, University of Michigan, 1966.

5. C. deBOOR \& B. SWARTZ, "Collocation at Gaussian points," SIAM J. Numer. Anal., v. 10, 1973, pp. 582-606.

6. J. DOUGLAS, JR. \& T. DUPONT, “Galerkin methods for parabolic equations," SIAM J. Numer. Anal., v. 7, 1970, pp. 575-626. MR 43 \#2863.

7. J. DOUGLAS, JR. \& T. DUPONT, "A finite element collocation method for quasilinear parabolic equations," Math. Comp., v. 27, 1973, pp. 17-28. 
8. J. DOUGLAS, JR. \& T. DUPONT, "Superconvergence for Galerkin methods for the two point boundary problem via local projections," Numer. Math., v. 21, 1973, pp. 270-278.

9. J. DOUGLAS, JR. \& T. DUPONT, "Galerkin approximations for the two point boundary problem using continuous, piecewise polynomial spaces," Numer. Math., v. 22, 1974, pp. 99-109.

10. J. DOUGLAS, JR., T. DUPONT, H. H. RACHFORD, JR. \& M. F. WHEELER, " $H^{-1}$ Galerkin methods for problems involving several space variables." (To appear.)

11. J. DOUGLAS, JR., T. DUPONT \& M. F. WHEELER, "An $L^{\infty}$ estimate and a superconvergence result for a Galerkin method for elliptic equations based on tensor products of piecewise poly nomials," Rev. Française Automatique Informatique et Recherche Operationnelle, v. R-2, 1974, pp. 61-66.

12. J. DOUGLAS, JR., T. DUPONT \& M. F. WHEELER, " $H^{1}$-Galerkin methods for the Laplace and heat equations," Mathematical Aspects of Finite Elements in Partial Differential Equations, C. deBoor (editor), Academic Press, New York, 1974.

13. T. DUPONT, "Galerkin methods for first order hyperbolics: An example," SIAM J. Numer. Anal., v. 10, 1973, pp. 890-899. MR 50 \#1540.

14. T. DUPONT, "Some $L^{2}$ error estimates for parabolic Galerkin methods," The Mathematical Foundations of the Finite Element Method with Applications to Partial Differential Equations, A. K. Aziz (editor), Academic Press, New York, 1972, pp. 491-504.

15. M. A. KRASNOSEL'SKIII, Topological Methods in the Theory of Nonlinear Integral Equations, GITTL, Moscow, 1956; English transl., Macmillan, New York, 1964. MR 20 \#3464; 28 \#2414.

16. M. A. KRASNOSEL'SKIĬ, G. M. VAÍNIKKO, P. P. ZABREǏKO, Ja. B. RUTICKIĬ \& V. Ja. STECENKO, Approximate Solution of Operator Equations, "Nauka", Moscow, 1969; English transl., Wolters-Noordhoff, Groningen, 1972. MR 41 \#4271.

17. P. LESAINT, "Finite element methods for symmetric hyperbolic equations," Numer. Math.,v. 21, 1973/74, pp. 244-255. MR 49 \#6648.

18. T. R. LUCAS \& G. W. REDDIEN, "A high order projection method for nonlinear two point boundary value problems," Numer. Math., v. 20, 1973, pp. 257-270.

19. M. S. MOCK, "A global a posteriori error estimate for quasilinear elliptic problems," Numer. Math., v. 24, 1975, pp. 53-61.

20. M. S. MOCK, "Explicit finite element schemes for first order symmetric hyperbolic systems," Numer. Math. (To appear.)

21. J. NITSCHE, "Ein Kriterium für die Quasi-Optimalität des Ritzschen Verfahrens," Numer. Math., v. 11, 1968, pp. 346-348. MR 38 \#1823.

22. H. H. RACHFORD, JR. \& M. F. WHEELER, "An $H^{-1}$ Galerkin procedure for the twopoint boundary value problem," Mathematical Aspects of Finite Elements in Partial Differential Equations, C. deBoor (editor), Academic Press, New York, 1974.

23. R. D. RUSSELL \& L. F. SHAMPINE, "A collocation method for boundary value problems," Numer. Math., v. 19, 1972 , pp. 1-28. MR 46 \#737.

24. M. H. SCHULTZ, " $L 2$ error bounds for the Raleigh-Ritz-Galerkin method," SIAM J. Numer. Anal., v. 8, 1971, pp. 737-748. MR 45 \#7967.

25. G. STRANG \& G. J. FIX, An Analysis of the Finite Element Method, Prentice-Hall, Englewood Cliffs, N. J., 1973.

26. V. THOMEE, "Spline approximation and difference schemes for the heat equation," The Mathematical Foundations of the Finite Element Method with Applications to Partial Differential Equations, A. K. Aziz (editor), Academic Press, New York, 1972, pp. 711-746.

27. V. THOMÉE \& L. WAHLBIN, “On Galerkin methods in semilinear parabolic problems," SIAM J. Numer. Anal., v. 12, 1975, pp. 378-389.

28. V. THOMÉE \& B. WENDROFF, "Convergence estimates for Galerkin methods for variable coefficient initial value problems," SIAM J. Numer. Anal., v. 11, 1974, pp. 1059-1068.

29. M. YAMAGUTI \& T. NOGI, "An algebra of pseudo difference schemes and its application," Publ. Res. Inst. Math. Sci. Ser. A, v. 3, 1967/68, pp. 151-166. MR 37 \#1762. 